\title{
Kondo effect in a quantum dot side-coupled to a topological superconductor
}

\author{
Minchul Lee, ${ }^{1}$ Jong Soo Lim, ${ }^{2}$ and Rosa López ${ }^{2,3}$ \\ ${ }^{1}$ Department of Applied Physics, College of Applied Science, Kyung Hee University, Yongin 446-701, Korea \\ ${ }^{2}$ Institut de Física Interdisciplinar i de Sistemes Complexos IFISC (CSIC-UIB), E-07122 Palma de Mallorca, Spain \\ ${ }^{3}$ Departament de Física, Universitat de les Illes Balears, E-07122 Palma de Mallorca, Spain
}

(Received 19 October 2012; published 12 June 2013)

\begin{abstract}
We investigate the dynamical and transport features of a Kondo dot side coupled to a topological superconductor (TS). The Majorana fermion states (MFSs) formed at the ends of the TS are found to be able to alter the Kondo physics profoundly: For an infinitely long wire where the MFSs do not overlap $\left(\epsilon_{m}=0\right)$ a finite dot-MFS coupling $\left(\Gamma_{m}\right)$ reduces the unitary-limit value of the linear conductance by exactly a factor $3 / 4$ in the weak-coupling regime ( $\Gamma_{m}<T_{K}$ ), where $T_{K}$ is the Kondo temperature. In the strong-coupling regime ( $\Gamma_{m}>T_{K}$ ), on the other hand, the spin-split Kondo resonance takes place due to the MFS-induced Zeeman splitting, which is a genuine many-body effect of the strong Coulomb interaction and the topological superconductivity. We find that the original Kondo resonance is fully restored once the MFSs are strongly hybridized $\left(\epsilon_{m}>\Gamma_{m}\right)$. This unusual interaction between the Kondo effect and the MFS can thus serve to detect the Majorana fermions unambiguously and quantify the degree of overlap between the MFSs in the TS.
\end{abstract}

DOI: 10.1103/PhysRevB.87.241402

PACS number(s): 73.63.-b, 73.21.La, 75.30.Hx

Introduction. One of the most paradigmatic effects in condensed matter physics is the celebrated Kondo effect. The ground state of a metal that contains magnetic impurities consists of a many-body spin singlet state where the localized impurities are entangled with the conducting states. ${ }^{1}$ The Kondo effect has been observed in manufactured nanostructures such as quantum dots (QDs), ${ }^{2-7}$ carbon nanotubes, ${ }^{8-10}$ nanowires, ${ }^{11}$ and so on. The great advantage of the observation of the Kondo effect in artificial setups is its high tunability and control by external and internal means. The Kondo physics can be further modified by utilizing different types of contact materials, say, superconducting ${ }^{12-14}$ or ferromagnetic ones. ${ }^{15-18}$ In particular, contacted with an $s$-wave superconductor, the Kondo spin experiences a quantum phase transition from the spin singlet state to the doublet state as the superconducting gap $\Delta$ increases over the Kondo temperature $T_{K} \cdot{ }^{12,13}$

Recently, topological superconductors (TSs) have attracted a lot of attention because they realize topological phases that support Majorana fermion states (MFSs) at their boundaries and at topological defects. ${ }^{19-24}$ The $p$-wave pairing is indispensable in hosting MFSs, and it has been known that the $p$-wave-like pairing can be induced in topological insulators ${ }^{21}$ and in semiconductors with strong spin-orbit coupling when brought into proximity with an $s$-wave superconductor. ${ }^{22-24}$ The MFSs formed at its boundaries are expected to be detected by attaching quantum objects to them and probing the interaction between them. Therefore, it is quite natural to think about intricate scenarios by combining Kondo-like artificial impurities with localized MFSs hosted in TSs. For example, the quantum impurity coupled to Majorana fermion (MF) edge channels of a two-dimensional helical TS is found to exhibit non-Fermi-liquid transport properties. ${ }^{25-27}$ The zero-bias conductance through a normal metal-QD-TS junction is found to be split when the MFS coupling exceeds the temperature. ${ }^{28}$

In order to probe the interplay between Kondo and Majorana physics unequivocally by means of usual transport measurements, we first choose a one-dimensional TS to host MFSs at its ends: a superconducting nanowire, ${ }^{22-24}$ the setup which recent experiments have used for the detection of Majorana edge states. ${ }^{29,30}$ Second, the one end of the TS is side coupled to the QD (Ref. 31) by a tunneling junction. ${ }^{32-34}$ The Kondo correlations are developed by attaching two normal contacts to the QD. We examine thoroughly the interplay between Kondo and Majorana physics by considering all the parameter regimes beyond the deep Kondo regime. ${ }^{28}$ Our findings indicate that the Kondo physics is dramatically altered depending on the relative strength of (i) the overlap of MFSs $\left(\epsilon_{m}\right)$, (ii) the dot-MFS coupling $\left(\Gamma_{m}\right)$, and (iii) the Kondo temperature $\left(T_{K}\right)$. In the sufficiently long wire case $\left(\epsilon_{m}=0\right)$, if the dot-MFS coupling is weak enough $\left(\Gamma_{m}<T_{K}\right)$, the destructive interference effect between paths that involve the MFS and the QD simultaneously or only the QD yields the appearance of an anti-Fano resonance with half-fermionic nature. Consequently, the linear conductance $G$ is reduced to a quantized value of $3 e^{2} / 2 h$, which unambiguously proves the existence of the MFS. This fractionally quantized conductance is quite distinguished from the similar setup using the side coupling to fermionic excitations $^{35-38}$ in which a perfect antiresonance $(G=0)$ or imperfect screening $(0<G<1)$ are expected. In addition, the quantized value is robust against small perturbations of parameters in contrast to the noninteracting counterpart ${ }^{31}$ since the Kondo resonance level always aligns with the MFS at the Fermi level. For the strong dot-MFS coupling $\left(\Gamma_{m}>T_{K}\right)$, the Kondo resonance becomes split due to the MFS-induced Zeeman splitting, which is also a genuine many-body effect of the strong Coulomb interaction and the topological superconductivity. Once the wire is short and the MFSs are strongly hybridized $\left(\epsilon_{m}>\Gamma_{m}\right)$, the dot-MFS coupling becomes less effective and the Kondo effect is restored. Thus, the interplay between the Kondo effect and the MFS could be an effective tool to unambiguously detect MFS and quantify the degree of overlap between the MFSs hosted in TSs.

Model. Our system is mapped onto a modified twofold degenerate Anderson model where the QD state is coupled to two normal-metal contacts and a topological superconducting 


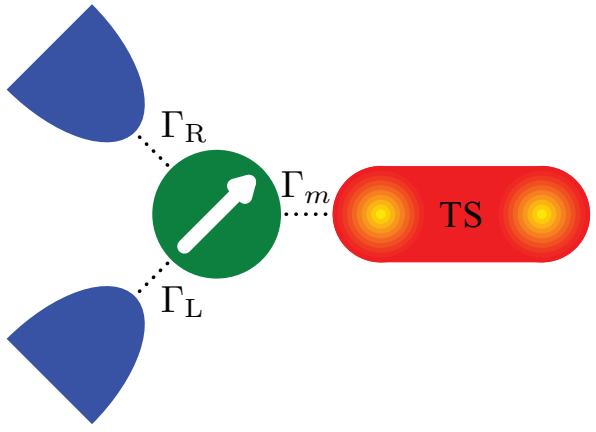

FIG. 1. (Color online) Quantum dot system coupled to two normal-metal leads (by tunneling rates $\Gamma_{L(R)}$ ) and to one end of a floating topological superconductor (by dot-MFS tunneling rate $\Gamma_{m}$ ). Due to the helical property of the MFS, only one of the spin components (say spin- $\downarrow$ ) is coupled to the MFS.

wire that hosts a pair of MFSs at its ends (see Fig. 1). We describe the TS with a low-energy effective model where the two MFSs are represented by the MF operators $\gamma_{i}(i=1,2) .{ }^{19}$ The operators follow the Clifford algebra $\left\{\gamma_{i}, \gamma_{j}\right\}=\delta_{i j}$, where $\gamma_{i}=\gamma_{i}^{\dagger}$. In terms of ordinary fermionic operator $f$, they can be written as $\gamma_{1}=\left(f+f^{\dagger}\right) / \sqrt{2}$ and $\gamma_{2}=\left(f-f^{\dagger}\right) / i \sqrt{2}$. In finite-length wires, the two MFSs have a finite overlap between their wave functions so that their coupling can lead a finite gap represented by $\epsilon_{m}$. Due to the helical property of such end states only one of the dot spin orientations (say spin- $\downarrow$ ) hybridizes with the MFS. ${ }^{32,39,40}$ Due to the macroscopic size of the TS we can safely neglect Coulomb charging effects, and the TS is floating in a sense that it is capacitively connected to a gate and no dc current flows through it. After these considerations, the Hamiltonian reads

$$
\begin{aligned}
\mathcal{H}= & \sum_{\ell \mathbf{k} \mu} \epsilon_{\mathbf{k}} c_{\ell \mathbf{k} \mu}^{\dagger} c_{\ell \mathbf{k} \mu}+\sum_{\mu} \epsilon_{d} d_{\mu}^{\dagger} d_{\mu}+U n_{\uparrow} n_{\downarrow}+2 i \epsilon_{m} \gamma_{1} \gamma_{2} \\
& +t_{m}\left(d_{\downarrow}^{\dagger} \gamma_{1}+\gamma_{1} d_{\downarrow}\right)+\sum_{\ell \mathbf{k} \mu}\left[t_{\ell} d_{\mu}^{\dagger} c_{\ell \mathbf{k} \mu}+(\text { H.c. })\right],
\end{aligned}
$$

where $c_{\ell \mathbf{k} \mu}^{\dagger}$ creates an electron with momentum $\mathbf{k}$, energy $\epsilon_{\mathbf{k}}$, and spin $\mu$ in the $\ell=L, R$ reservoir. Two normal contacts share a same flat-band structure with a half bandwidth $D$ and density of states $\rho$. The operator $d_{\mu}^{\dagger}$ creates an electron with spin $\mu$ in dot, and $n_{\mu}=d_{\mu}^{\dagger} d_{\mu}$ is the dot occupation for spin $\mu$. We focus on the case of single orbital level with energy $\epsilon_{d}$ and strong Coulomb interaction denoted as $U$. In our setup the QD should be spin degenerate. ${ }^{34}$ The critical magnetic field $(\sim 0.1 \mathrm{meV})^{29,30}$ required to induce $p$-wave superconductivity in the TS wire is not so strong as to destroy the Kondo effect due to the small $g$ factor in QDs. The superfluous magnetic field can even be eliminated by using proximity with a magnetic insulator to induce the Zeeman splitting in the TS wire. ${ }^{21,41}$ The dot electron hybridizes (i) with the conduction electrons in the contacts with a tunneling amplitude $t_{\ell}$, and (ii) with the nearest MFS with a tunneling amplitude $t_{m}$. Both couplings define the two tunneling rates: $\Gamma_{\ell}=\pi \rho t_{\ell}^{2}$ and $\Gamma_{m}=\pi \rho_{\mathrm{dot}} t_{m}^{2}$. Throughout our study, we focus on the Kondo regime, $\epsilon_{d}<\epsilon_{F}=0<\epsilon_{d}+U$ and $\Gamma \equiv \Gamma_{L}+\Gamma_{R} \ll\left|\epsilon_{d}\right|, \epsilon_{d}+U$ at zero temperature, where $\epsilon_{F}$ is the Fermi energy. For a nonperturbative study of the many-body effect, we adopt the well-known numerical renormalization group (NRG) method: ${ }^{42-44}$ One can refer to Ref. 45 for a review. For the analysis, we calculate the spectral densities $A_{\mu(i)}=\sum_{n}\left|\left\langle n\left|d_{\mu}^{\dagger}\left(\gamma_{i}\right)\right| 0\right\rangle\right|^{2} \delta\left(\omega-E_{n}+E_{0}\right)$ and $A_{z z}=$ $\sum_{n}\left|\left\langle n\left|S_{z}\right| 0\right\rangle\right|^{2} \delta\left(\omega-E_{n}+E_{0}\right)$, where $|n\rangle$ is the many-body eigenstate with energy $E_{n}$ and $|0\rangle$ is the ground state. From the spin-resolved spectral densities the transmission through the dot can be obtained, $T(\omega)=2 \pi \Gamma_{L} \Gamma_{R} /\left(\Gamma_{L}+\Gamma_{R}\right) \sum_{\mu} A_{\mu}(\omega)$, and the linear conductance is $G=\left(2 e^{2} / h\right) T(\omega=0)$.

Noninteracting case $(U=0)$. Before addressing the full system we examine a simple case. In the noninteracting case, the effect of the MFS on the transport can be handled by using the spinless model since the MFS is coupled to spin- $\downarrow$ electrons only. ${ }^{31}$ Therefore, $A_{\uparrow}(\omega)$ is not affected by the MFS. On the other hand, $A_{\downarrow}(\omega)$ features the destructive interference between the spin- $\downarrow$ electron and the MFS. In the on-resonant case $\left(\epsilon_{d}=\epsilon_{m}=0\right)$ and for small $t_{m}$, the resultant anti-Fano resonance leads to a half-dip at $\omega=0: \pi \Gamma A_{\downarrow}(0)$ is reduced from 1 to $1 / 2$. The dip width is comparable with the resonance width $\Gamma_{m}$ in $A_{1}(\omega)$, which is numerically found to be $\Gamma_{m} \approx$ $\pi \rho_{\mathrm{dot}} t_{m}^{2}$ with $\rho_{\mathrm{dot}}=1 / \Gamma$. As $t_{m}$ increases further $\left(\Gamma_{m}>\Gamma\right)$, $A_{\downarrow}(\omega)$ develops two side peaks at $\omega \sim \pm \sqrt{2} t_{m}$ which come from the hybridization between the spin- $\downarrow$ dot electron and the MFS, while $\pi \Gamma A_{\downarrow}(0)=1 / 2$ is maintained due to the zeroenergy MFS. ${ }^{31}$ Consequently, the linear conductance $G$ at zero temperature is quantized to $e^{2} / h+e^{2} / 2 h=3 e^{2} / 2 h$ as long as $\epsilon_{m}=0$, which signals the presence of MFSs in noninteracting side-coupled nanowire setups. Note that the exact fractional quantization happens exactly at the resonant condition $\left(\epsilon_{m}=\right.$ 0 ). Hence a fine tuning is necessary to observe the quantization in the noninteracting case.

Kondo regime, long wire $\left(\epsilon_{m}=0\right)$ case. In Fig. 2 we present our NRG results for the spin-resolved dot and MF

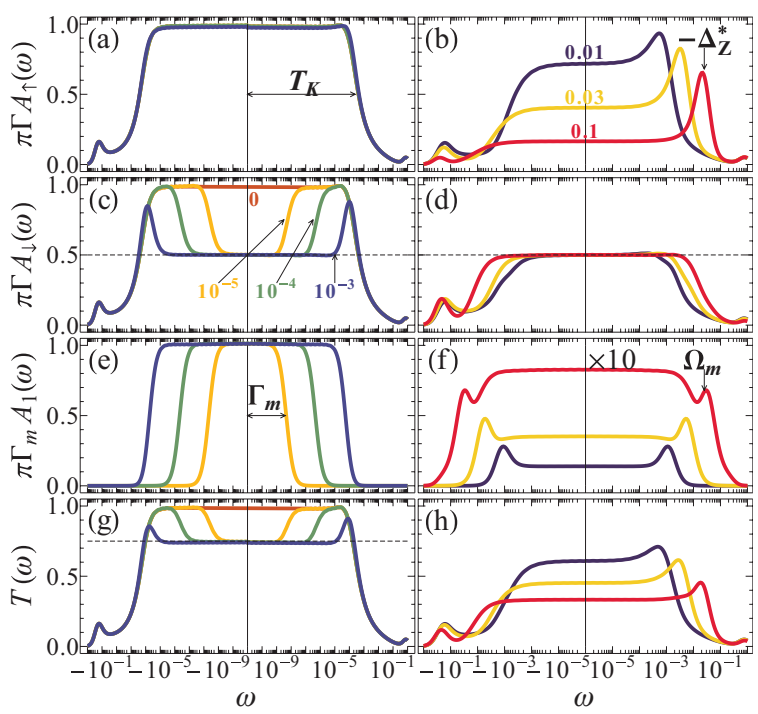

FIG. 2. (Color online) Dynamical and transport features for an infinitely long nanowire with $\epsilon_{m}=0$. Left and right panels represent the weak- $\left(\Gamma_{m}<T_{K}\right)$ and the strong-coupling $\left(\Gamma_{m}>T_{K}\right)$ regimes, respectively: [(a), (b)] dot spin- $\uparrow$ spectral density, [(c), (d)] dot spin- $\downarrow$ spectral density, [(e), (f)] $\gamma_{1}$-operator spectral density, and [(g), (h)] transmission coefficient for annotated values of $t_{m}$. We have used $\epsilon_{d}=-0.2, U=1, \Gamma_{L}=\Gamma_{R}=0.02, D=1$, and $\Gamma_{m}=2 t_{m}^{2} / \Gamma$. 
spectral densities in the $\epsilon_{m}=0$ case. For the trivial case of $t_{m}=0$ the dot spin-resolved spectral density $A_{\mu}(\omega)$ features a Kondo resonance peak centered at $\omega=0$ with a width $T_{K}: T_{K}=\sqrt{\Gamma U / 2} \exp \left[\pi \epsilon_{d}\left(\epsilon_{d}+U\right) /(2 \Gamma U)\right] .{ }^{46}$ For a finite but still weak coupling $\left(T_{K}>\Gamma_{m}\right)$, the physics resembles that of the noninteracting case: $A_{\uparrow}(\omega)$ [see Fig. 2(a)] is not altered by the presence of the MFS and is kept intact. The Kondo resonance peak at $\omega=0$ and two mean-field peaks at $\omega \approx \epsilon_{d}, \epsilon_{d}+U$ remain quite independent of the dot-MFS coupling $t_{m}$. The half-fermionic anti-Fano resonance due to the side-coupled MFS leads to a half-dip in $A_{\downarrow}(\omega)$ [see Fig. 2(c)] whose width is the same as the width $\Gamma_{m}$ of the Lorentzian-like resonance peak of $A_{1}(\omega)$ [see Fig. 2(e)]. Therefore, the total transmission coefficient $T(\omega)$ illustrated in Fig. 2(g) exhibits a Kondo peak with a dip so that $T(\omega=0)=3 / 4$ producing a linear conductance $G=3 e^{2} / 2 h$. The low-energy physics in the Kondo effect is usually understood in terms of a noninteracting model: a resonant level at $\epsilon_{d}^{*}=0$ with a width $T_{K}$. The observed features above might be predictable in this noninteracting frame. However, one should be very cautious in using the effective theory since $t_{m}$ is found to be strongly renormalized. We numerically found that $\Gamma_{m}=\pi \rho_{\mathrm{dot}}^{*} t_{m}^{* 2} \approx$ $2 t_{m}^{2} / \Gamma$. Noting that $\rho_{\text {dot }}^{*} \sim 1 / T_{K}$ in the Kondo regime, the renormalization should lead to $t_{m}^{*} \sim t_{m} \sqrt{T_{K} / \Gamma}$ for $T_{K}>\Gamma_{m}$. The many-body correlations not only produce the Kondo effect but also renormalize the dot-MFS coupling strongly. It should be noted that the exotic quantization in the noninteracting case happens only when the QD resonant level aligns with the MFS level, which requires a fine tuning of the QD parameters. ${ }^{31}$ In the Kondo case, this requirement is satisfied without fine tuning since the Kondo level is always pinned at the Fermi level. Hence the quantization in the Kondo regime is quite robust against small perturbations in QD parameters.

Let us now move to the strong-coupling regime $\left(\Gamma_{m}>T_{K}\right)$ (see the right panel of Fig. 2). Here, the dot-MFS coupling dominates over the Kondo effect. Strikingly, the Kondo effect survives the strong interference from the side coupling, and the effect of the dot-MFS coupling is quite different for two spins. The central peak in $A_{\downarrow}(\omega)$ [see Fig. 2(d)] remains at $\omega=0$ but gets wider with $t_{m}$, while $\pi \Gamma A_{\downarrow}(\omega=0)$ is pinned at $1 / 2$. Its width is in par with that of the central peak in $A_{1}(\omega)$ [see Fig. 2(f)], reflecting the coupling between the MFS and the spin- $\downarrow$ dot electron. Interestingly, the peak of $A_{\uparrow}(\omega)$ [see Fig. 2(b)] shifts and gets wider with increasing $t_{m}$ : The peak moves toward positive (negative) frequencies in the hole (electron) -dominant regime, $\delta \equiv 2 \epsilon_{d}+U>0$ $(\delta<0)$. In the particle-hole symmetry point, the Kondo peak in $A_{\uparrow}(\omega)$ remains at $\omega=0$ and only gets broader. This behavior reminds us of the usual Zeeman-splitting-induced shift of the Kondo peak. In fact, by diagonalizing the interacting QD-MFS system decoupled from the leads $\left(t_{\ell}=0\right)$, or by applying the Schrieffer-Wolff transformation, we found that the dot-MFS coupling induces a finite effective Zeeman splitting on dot spins. ${ }^{40}$ For $t_{m} \ll \epsilon_{d}+U,\left|\epsilon_{d}\right|$, the induced Zeeman splitting is given by

$$
\Delta_{Z}=-\frac{t_{m}^{2}}{2} \frac{\delta}{\left(\epsilon_{m}+\delta-\epsilon_{d}\right)\left(\epsilon_{m}-\epsilon_{d}\right)} .
$$

This is the combined effect of (i) the coupling to the MFS with the spin- $\downarrow$ dot electrons and (ii) the Coulomb interaction,

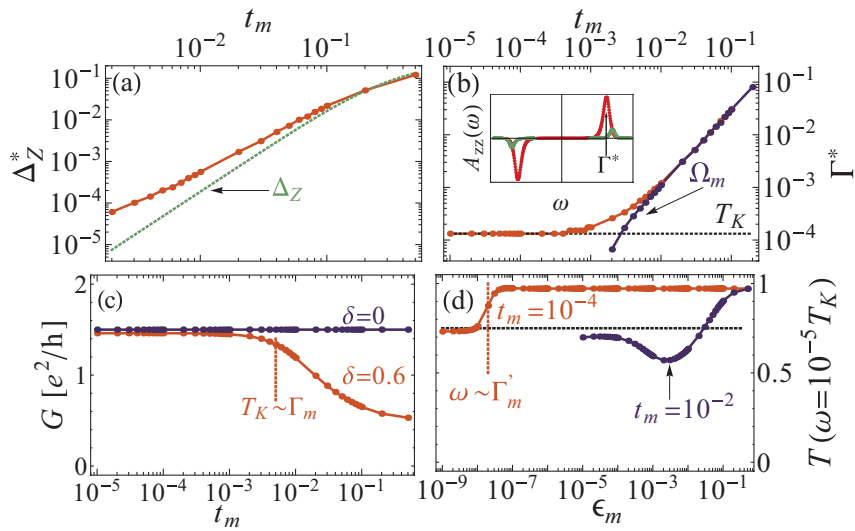

FIG. 3. (Color online) (a) $\Delta_{Z}$ and $\Delta_{Z}^{*}$ (in the strong-coupling regime) at $\epsilon_{m}=0$ and (b) $\Gamma^{*}$ and $\Omega_{m}$ as functions of $t_{m}$. Inset: typical shape of $A_{z z}(\omega)$. (c) Linear conductance $G$ versus $t_{m}$ at $\epsilon_{d}=-0.2(\delta=0.6)$ and $\epsilon_{d}=-0.5(\delta=0)$. (d) Transmission coefficients versus $\epsilon_{m}$ at finite frequency $\omega=10^{-5} T_{K}$ for $t_{m}=10^{-4}$ (weak-coupling regime) and $t_{m}=10^{-2}$ (strong-coupling regime). We have used the same values as used in Fig. 2.

and it is one of our key results. Remarkably, $\Delta_{Z}$, having the sign opposite to $\delta$, vanishes at the particle-hole symmetry point $(\delta=0)$ since it is generated by dot charge fluctuations in analogy to the exchange field induced by ferromagnetic contacts attached to an interacting QD. ${ }^{16,18}$ The appearance of the finite Zeeman splitting is not surprising since the time-reversal symmetry is already broken in inducing the $p$-wave pairing in the TS wire. Upon coupling the dot to normal leads, $\Delta_{Z}$ becomes renormalized to $\Delta_{Z}^{*}$, which is larger than $\Delta_{Z}$ : We have confirmed it by applying Haldane's scaling theory. ${ }^{47}$ The peak position in $A_{\uparrow}(\omega)$ is then identified as $\Delta_{Z}^{*}$ and plotted in Fig. 3(a). We observed a strong renormalization of $\Delta_{Z}$ for smaller $t_{m}$. In parallel with the shift of the Kondo peak of $A_{\uparrow}(\omega)$, the peak of $T(\omega)$ [see Fig. 2(h)] moves with $t_{m}$ away from $\omega=0$, and accordingly its value at $\omega=0$ decreases. The linear conductance then decreases with $t_{m}$ for $\Gamma_{m}>T_{K}$. However, it saturates to $e^{2} / 2 h$ at larger values of $t_{m}$ since $A_{\downarrow}(\omega=0)$ remains unchanged. In the particle-hole symmetric case $\left(\Delta_{Z}^{*}=0\right)$, the Kondo peak of $A_{\uparrow}$ is pinned at $\omega=0$ so that the linear conductance is fixed to $3 e^{2} / 2 h$, independent of $t_{m}$ [see Fig. 3(c)].

The spin susceptibility $A_{z z}(\omega)$ helps us to know about the influence of the MFS on the spin correlation. As shown in Fig. 3(b), $A_{z z}(\omega)$ has two peaks at $\omega= \pm \Gamma^{*}$. These peaks arise because the spin fluctuations are enhanced by breaking the spin correlations. Hence, $\Gamma^{*}$ should be related to the spin binding energy. We found that $\Gamma^{*}=T_{K}$ in the weak-coupling regime $\left(T_{K}>\Gamma_{m}\right)$, reflecting the presence of Kondo correlations. In the strong-coupling regime $\left(T_{K}<\Gamma_{m}\right)$ we found $\Gamma^{*} \approx \Omega_{m}$, i.e., the side peak position of $A_{1}(\omega)$ [see Fig. 2(f)]. In this case, the spin- $\downarrow$ is more strongly hybridized with the MFS so that its correlation energy $\Omega_{m}$ defines the relevant spin binding energy. Note that the Kondo effect remains nonetheless.

Kondo regime, short wire $\left(\epsilon_{m} \neq 0\right)$ case. In real experiments the finite size of the wire makes the two MFSs overlap, always giving rise to a finite $\epsilon_{m} \neq 0$ or the energy splitting between two fermionic levels $|0\rangle$ and $|1\rangle \equiv f^{\dagger}|0\rangle$. For large overlap $\left(\left|\epsilon_{m}\right| \gtrsim \Gamma_{m}\right)$, the fermionic $f$ levels at energy $\pm \epsilon_{m}$ do not 


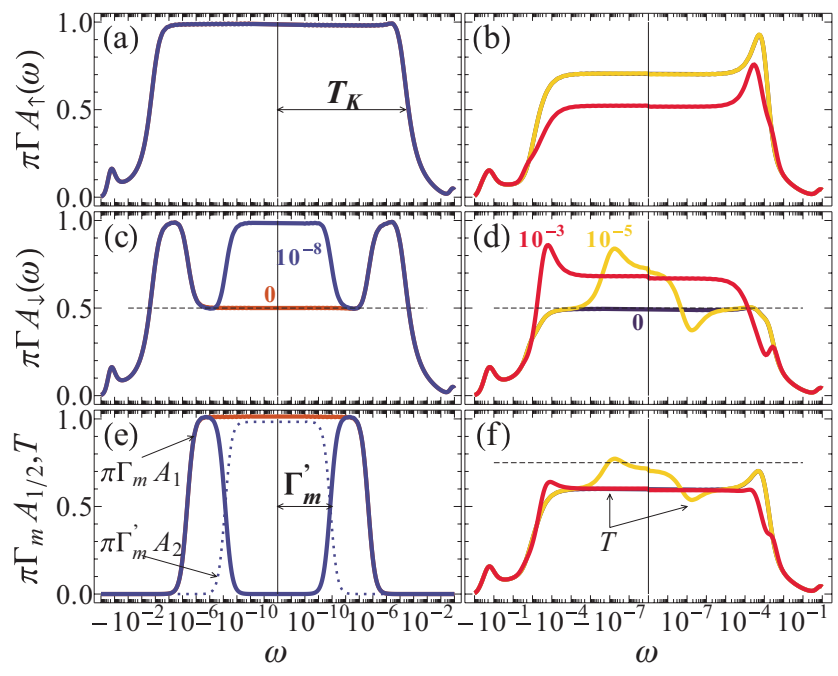

FIG. 4. (Color online) Dynamical features for a realistic wire for small overlaps $0<\epsilon_{m}<\Gamma_{m}$. Left and right panels represent the weak coupling regime $\left(t_{m}=10^{-4}, \Gamma_{m}<T_{K}\right)$ and the strong coupling regime $\left(t_{m}=10^{-2}, \Gamma_{m}>T_{K}\right)$, respectively: (a), (b) dot spin$\uparrow$ spectral density, (c), (d) dot spin- $\downarrow$ spectral density, (e) $\gamma_{1,2}$-operator spectral density, (f) transmission coefficient for annotated values of $\epsilon_{m}$. The dotted line in (e) represents $\pi \Gamma_{m}^{\prime} A_{2}(\omega)$. We have used the same values as used in Fig. 2 for other parameters.

interfere with the Kondo resonant level formed at the Fermi level any longer so that the Kondo physics is completely restored, irrespectively of $t_{m} \cdot{ }^{40}$ Figure $3(\mathrm{~d})$ shows that the transmission coefficient recovers the Kondo value at large value of $\epsilon_{m}$.

Now consider the case of small overlap $\left(\epsilon_{m}<\Gamma_{m}\right)$. For finite $\epsilon_{m}, \gamma_{2}$ is coupled to $\gamma_{1}$ with coupling strength $2 \epsilon_{m}$. The spectral density $A_{2}(\omega)$, being $\delta(\omega)$ for $\epsilon_{m}=0$, now forms a Lorentzian-like distribution broadened by the $\gamma_{1}-\gamma_{2}$ coupling [see the dotted lines in Fig. 4(e)]. Since the density of states of $\gamma_{1}$ is $1 / \Gamma_{m}$ due to the QD-MFS hybridization, the width of the broadening of $A_{2}$ should be $\Gamma_{m}^{\prime} \approx\left(2 \epsilon_{m}\right)^{2} / \Gamma_{m}$. The $\gamma_{1}-\gamma_{2}$ coupling in turn invokes another anti-Fano resonance in $A_{1}$ : In the weak-coupling regime, $A_{1}$ [see Fig. $4(\mathrm{e})$ ] is found to have a dip at $\omega=0$ whose width is given by $\Gamma_{m}^{\prime}$. The scattering from the resonance level of $\gamma_{2}$ leads to the destructive interference: Since both $\gamma_{1}$ and $\gamma_{2}$ are half-fermionic, the destructive interference is perfect. The dip in $A_{1}$ accordingly affects $A_{\downarrow}$ [see Fig. 4(c)], while $A_{\uparrow}$ [see Fig. 4(a)] is not so affected. $A_{\downarrow}$ displays a three-peak structure with a central peak of width $\sim \Gamma_{m}^{\prime}$. The peak signals the disappearance of the anti-Fano resonance by the MFS at the Fermi level. As $\epsilon_{m}$ grows up to values close to $\Gamma_{m}$ the three peaks coalesce into a single resonance, restoring the Kondo physics. The transmission coefficient $T(\omega)$ would then show a steplike increase from $3 e^{2} / 2 h$ to $2 e^{2} / h$ at $\omega \sim \Gamma_{m}^{\prime}$ with increasing $\epsilon_{m}$ [see Fig. 3(d)].

In the strong-coupling regime $\left(T_{K}<\Gamma_{m}\right)$ the finite $\epsilon_{m}$ abolishes the half-fermionic Fano resonance at the Fermi level as well: $\pi \Gamma A_{\downarrow}(\omega=0)$ is not pinned to $1 / 2$ but larger than $1 / 2$ for any finite value of $\epsilon_{m}$ [see Fig. 4(d)]. More precisely, the half-value pinning is retained only in a range of frequency, $\Gamma_{m}^{\prime}<|\omega|<\Gamma_{m}$ for $\epsilon_{m}<\Gamma_{m}$. In addition, the large $f$-level splitting adds an additional peak at $\omega \approx \mp \Gamma_{m} / \pi$ for $\delta \gtrless 0$ and a small dip in the opposite side. The peak moves toward the higher frequencies with increasing $\epsilon_{m}$ until $\epsilon_{m} \sim \Gamma_{m}$ and eventually returns to the Kondo peak as the Kondo physics is revived. The effective Zeeman splitting encountered in $A_{\uparrow}$ also diminishes with increasing $\epsilon_{m}$ [see Eq. (2)]: The peak gradually moves toward $\omega=0$. Hence, in contrast to the weak-coupling regime, the restoration of the linear conductance to the Kondo value is rather slow so that the full recovery is obtained for $\epsilon_{m} \gg \Gamma_{m}$ [see Fig. 3(d) for the transmission coefficient].

Conclusions. We have investigated the effect of the MFS on the Kondo physics in the side-coupled geometry. Even though the MFS is based on the superconductivity which would suppress the Kondo effect, it is found that the Kondo effect survives the interaction with the MFS in a modified form. We found that dot-MFS coupling introduces an electronic way to control the effective Zeeman splitting. In addition, our results show that the energy-resolved transmission through the dot provides an excellent way to detect the MFS in a much clearer way and to examine the properties of MFSs and the overlap between them.

Acknowledgments. We acknowledge S. Andergassen and R. Žitko for a critical reading of the manuscript and useful discussion. M.L. was supported by the Center for Academic Computing at Kyung Hee University for computer resources and the National Research Foundation grant funded by the Korea MEST (Grant No. 2011-0030790). R.L. and J.S.L. were supported by MINECO Grants No. FIS2011-2352 and No. CSD2007-00042 (CPAN).

\footnotetext{
${ }^{1}$ A. Hewson, The Kondo Problem to Heavy Fermions (Cambridge University Press, New York, 1993).

${ }^{2}$ L. P. Kouwenhoven, T. H. Oosterkamp, M. W. S. Danoesastro, M. Eto, D. G. Austing, T. Honda, and S. Tarucha, Science 278, 1788 (1997).

${ }^{3}$ D. Goldhaber-Gordon, J. Göres, M. A. Kastner, H. Shtrikman, D. Mahalu, and U. Meirav, Phys. Rev. Lett. 81, 5225 (1998).

${ }^{4}$ D. Goldhaber-Gordon, H. Shtrikman, D. Mahalu, D. AbuschMagder, U. Meirav, and M. A. Kastner, Nature (London) 391, 156 (1998).
}

${ }^{5}$ S. M. Cronenwett, T. H. Oosterkamp, and L. P. Kouwenhoven, Science 281, 540 (1998).

${ }^{6}$ J. Schmid, J. Weis, K. Eberl, and K. von Klitzing, Physica B: Condensed Matter 256, 182 (1998).

${ }^{7}$ W. G. van der Wiel, S. D. Franceschi, T. Fujisawa, J. M. Elzerman, S. Tarucha, and L. P. Kouwenhoven, Science 289, 2105 (2000).

${ }^{8}$ J. Hygard, D. H. Cobden, and P. E. Lindelof, Nature (London) 408, 342 (2000).

${ }^{9}$ T. W. Odom, J.-L. Huang, C. L. Cheung, and C. M. Lieber, Science 290, 1549 (2000). 
${ }^{10}$ P. Jarillo-Herrero, J. Kong, H. S. J. van der Zant, C. Dekker, and L. P. Kouwenhoven, Nature (London) 434, 484 (2005).

${ }^{11}$ O. Klochan, A. P. Micolich, A. R. Hamilton, K. Trunov, D. Reuter, and A. D. Wieck, Phys. Rev. Lett. 107, 076805 (2011).

${ }^{12}$ M.-S. Choi, M. Lee, K. Kang, and W. Belzig, Phys. Rev. B 70, 020502(R) (2004).

${ }^{13}$ C. Karrasch, A. Oguri, and V. Meden, Phys. Rev. B 77, 024517 (2008).

${ }^{14}$ R. Žitko, M. Lee, R. López, R. Aguado, and M.-S. Choi, Phys. Rev. Lett. 105, 116803 (2010).

${ }^{15}$ J. Martinek, Y. Utsumi, H. Imamura, J. Barnas, S. Maekawa, J. Konig, and G. Schon, Phys. Rev. Lett. 91, 127203 (2003).

${ }^{16}$ M.-S. Choi, D. Sánchez, and R. López, Phys. Rev. Lett. 92, 056601 (2004).

${ }^{17}$ J. S. Lim, R. López, G. L. Giorgi, and D. Sánchez, Phys. Rev. B 83, 155325 (2011).

${ }^{18}$ R. Žitko, J. S. Lim, R. López, J. Martinek, and P. Simon, Phys. Rev. Lett. 108, 166605 (2012).

${ }^{19}$ A. Y. Kitaev, Phys.-Usp. 44, 131 (2001).

${ }^{20}$ N. Read and D. Green, Phys. Rev. B 61, 10267 (2000).

${ }^{21}$ L. Fu and C. L. Kane, Phys. Rev. Lett. 100, 096407 (2008).

${ }^{22}$ R. M. Lutchyn, J. D. Sau, and S. Das Sarma, Phys. Rev. Lett. 105, 077001 (2010).

${ }^{23}$ Y. Oreg, G. Refael, and F. von Oppen, Phys. Rev. Lett. 105, 177002 (2010).

${ }^{24}$ J. Alicea, Phys. Rev. B 81, 125318 (2010).

${ }^{25}$ R. Žitko, Phys. Rev. B 81, 241414(R) (2010).

${ }^{26}$ R. Žitko, Phys. Rev. B 83, 195137 (2011).

${ }^{27}$ R. Žitko and P. Simon, Phys. Rev. B 84, 195310 (2011).
${ }^{28}$ A. Golub, I. Kuzmenko, and Y. Avishai, Phys. Rev. Lett. 107, 176802 (2011).

${ }^{29}$ V. Mourik, K. Zuo, S. M. Frolov, S. R. Plissard, E. P. A. M. Bakkers, and L. P. Kouwenhoven, Science 336, 1003 (2012).

${ }^{30}$ A. Das, Y. Ronen, Y. Most, Y. Oreg, M. Heiblum, and H. Shtrikman, Nat. Phys. 8, 887 (2012).

${ }^{31}$ D. E. Liu and H. U. Baranger, Phys. Rev. B 84, 201308 (2011).

${ }^{32}$ K. Flensberg, Phys. Rev. B 82, 180516 (2010).

${ }^{33}$ M. Leijnse and K. Flensberg, Phys. Rev. B 84, 140501 (2011).

${ }^{34}$ M. Leijnse and K. Flensberg, Phys. Rev. Lett. 107, 210502 (2011).

${ }^{35}$ M. E. Torio, K. Hallberg, S. Flach, A. E. Miroshnichenko, and M. Titov, Eur. Phys. J. B 37, 399 (2004).

${ }^{36}$ A. A. Aligia and L. A. Salguero, Phys. Rev. B 70, 075307 (2004).

${ }^{37}$ M. Lee and C. Bruder, Phys. Rev. B 73, 085315 (2006).

${ }^{38}$ Y. Tanaka, N. Kawakami, and A. Oguri, Phys. Rev. B 85, 155314 (2012).

${ }^{39}$ D. Sticlet, C. Bena, and P. Simon, Phys. Rev. Lett. 108, 096802 (2012).

${ }^{40}$ See Supplemental Material at http://link.aps.org/supplemental/ 10.1103/PhysRevB.87.241402 for full details of the calculations.

${ }^{41}$ J. D. Sau, R. M. Lutchyn, S. Tewari, and S. Das Sarma, Phys. Rev. Lett. 104, 040502 (2010).

${ }^{42}$ K. G. Wilson, Rev. Mod. Phys. 47, 773 (1975).

${ }^{43}$ H. R. Krishna-murthy, J. W. Wilkins, and K. G. Wilson, Phys. Rev. B 21, 1003 (1980); 21, 1044 (1980).

${ }^{44}$ W. Hofstetter, Phys. Rev. Lett. 85, 1508 (2000).

${ }^{45}$ R. Bulla, T. A. Costi, and T. Pruschke, Rev. Mod. Phys. 80, 395 (2008).

${ }^{46}$ F. D. M. Haldane, J. Phys. C: Solid State Phys. 11, 5015 (1978).

${ }^{47}$ F. D. M. Haldane, Phys. Rev. Lett. 40, 416 (1978). 